\title{
Workflow model with version change based on task-relationships
}

\author{
Xuezi Yang \\ College of Computer Science and Technology, \\ Jilin University \\ ChangChun,China \\ yangxz13@mails.jlu.edu.cn
}

Xiaodong Zhu*

College of Computer Science and Technology, Jilin University

ChangChun,China

zhuxd@jlu.edu.cn

\author{
Siyuan Chen \\ College of Mechanical Science and Engineering, \\ Jilin University \\ ChangChun,China \\ @qq.com
}

\author{
Yuanning Liu \\ College of Computer Science and Technology, \\ Jilin University \\ ChangChun, China \\ lyn@jlu.edu.cn \\ Fei He \\ College of Computer Science and Technology, \\ Jilin University \\ ChangChun,China \\ 14753152@qq.com
}

\begin{abstract}
The version change, which cannot be considered as an independent process, is a fundamental factor for designing the workflow model. Due to that the traditional workflow model is unidirectional, it could not identify the range of version change and restart the tasks which are need to be changed. This paper proposes a workflow model, which is implemented using tree structure as basis. This model is based on task-relationships and takes version change as an influential factor for workflow management, by analyzing the attributes of tasks as well as the physical and logical relationships among tasks, it identifies proper ranges of version change of workflow without breaking data consistency, therefore it reduces the unnecessarily repeated task performing when version change happens, which helps achieving the goal of reducing project investment. Furthermore, this model helps to build strong workflow restarting mechanism which is more compatible for version change, thus workflow can be restarted and tasks can be performed efficiently after the ranges of version change are identified. In sum, this model ensures the controllability and spontaneity of workflow, which is useful for supporting workflow reliably and efficiently.
\end{abstract}

Keywords-Version change; Workflow model; style;Physical and logical relationship; Restart workflow(key words)

\section{INTRODUCTION}

Workflow Management Coalition(WFMC)defines the workflow as follow: an automation of a business process, in whole or part, during which documents, information or tasks are passed from one participant to another for action, according to a set of procedural rules [1]. With the development of science and technology, more and more companies make their work focus on to manage the data of product and to use the workflow to manage data and task. However, due to the rapid development of social and technical, the market environment has changed more and more quickly. Therefore, the production process will become more complex and comprehensive, the main features of the production process are presented: (1)the Greater mutation; (2)the greater uncertainty; (3)the greater complexity; (4)the greater rate of change; (5)the greater diversity; (6)the smaller stability; (7)the smaller identity; (8)the smaller ability of predict [2]. Trough the production process which have the higher degree of uncertainly and unpredictability, the conception of Engineering changes come forward. Engineering changes(ECs): Refers to change the product and its components of the form, assembly, material, size and function [3]. It can be a simple process of revision of the document, and it can also be a complex process of redesigning the products and manufacturing process. Studies have, in fact, shown that the engineering-change management(ECM) could represent from $70 \backslash \%$ to $80 \backslash \%$ of the final product' $\mathrm{s}$ costlcite $[4,5]$. In reality, the ECs is a norm rather than an exception in many product development firms. Consequently, ECM is a major competitive factor in product design and development process that should not be neglected [6].

The studies of the method of the ECs is focus on how to reduce the impact of engineering change on manufacturing engineering. The version change of engineering, mainly used in the process of the products which have experience the repeated verification and modification, is the a key breach of the method of the ECs. This paper will propose a workflow model, which is 
compatible with the version change of the nodes. The model make the production process mapping into the task node of workflow and consider the effect of version change on workflow.

\section{RELATED WORK}

The management of version change of workflow is different from general features of the management of the product data which focus on the management of product data relationship and the process management. Moreover, It is also different from the management of the workflow which focus on improving the efficiency of the process and strengthening the feedback strategy between the processes. In order to reduce the impact of the change and improve the efficiency of the change, it pays attention to that how to reuse the management of the product data and the management of the workflow and effective to implement the management of change. It is based on the workflow to determine a more appropriate change range, to reduce impact which is unnecessary for version change of workflow and to guarantee the data consistency which is key of version change of workflow.

At present, many flexible workflow engines focus mainly on the adjustments to business processes changes, and general workflow system can not be dynamically adjusted in real time when nodes' internal circuit changes, its version changes, or countersigned changes, it can not be fully adjusted according to changing needs [7]. In many previous studies on workflow model and workflow engine, the possibility of the version change of the workflow always be ignored. For example, the workflow engines in the paper of Liu etc [1]. In order to make the workflow more adaptive the development of product, they add the conception of product structure into the workflow engines. But they ignore that the product development is a complex process and some mistakes are unavoidable. This neglect means when we need to revise the task of the workflow, the only way is to restructure another workflow and to execute it from the first task. In fact, some of the tasks have no relevance to the node of version change. In the paper of $\mathrm{Yu}$ [7], it introduces a flexible workflow engines and consider the possibility of changing the workflow data. Nevertheless, in the process of version change of task, they did not take into account the relevance of workflow nodes of the processes, so that the change of range is greater than the range of the real need to change. And the bigger range not only lead to execute the task which is uncorrelated with the real version change task but also cause the unneces sary waste of human resources. Eventually, it can lead to decrease efficiency of production process.

Therefore, in this paper propose a model of workflow, namely TR-VC. It will focus on the point as follows:

The first one is how to reduce repetitive execute the unnecessary task and ensure the proper range of version change on the basis of the consistency of the data. The second one is how to guarantee the workflow can correct restart when include the task of version change.

\section{MODEL}

\section{A. The Definition of model}

In term of the analysis of the business needs of the version change, the analysis of node attributes and the analysis of workflow engine, the version changes of the workflow should involve two aspects:

Firstly, the aspect is how to determine the range of the change of the workflow. It mainly based on the point as follows:

- The reversibility of node, that is weather the data of the node can be reverse.

- The existence of node' $s$ data, that is, the change of data must based on the data which already existed.

- The mutual relationship between the nodes, that is include two aspects: one is the physical relationship, that is the data transition between the nodes; the other is the logical relationship, to consider the Promote relationship between workflow nodes and then abstract the logical relationship. In order to ensure data consistency of all the nodes, the nodes which have mutual relationship with the change node should be considered to change.

Secondly, the aspect is how to correct restart the workflow. There are two mainly points:

- The sequence of execute.

- The driver of statue.

It is based on the above factors, I can built the model of workflow to adjust the change of the nodes' data. At first, I need to define the conception as follow.

1) The nodes of TR-VC is present by a tuple (NUM,NN,NT,NA,IEV,NS,ND,NDP).

- NUM: present the nodes' number.

- NN: present the nodes' name.

- NT: present the nodes' type and NT $\in \mathrm{T}$.

- NA: present the application interface of the nodes.

- IEV: present that Whether the node has the ability to change.

- NS: present the status of the node and NS $\in$ status .

- ND: present the data of nodes.

- NDP: present that the direction of data flow. And the relationship of dependency be called the physical relationship.

2) The definition of logical dependency of collection $T=\{B, P, R n, L, S, t\}$.

- $\mathrm{B}($ branch node $)$ is a collection, $B=\{X 1$, $X 2 . ., X n\}, n \geqslant 2$.and there are two features:(1)if $X n$ $\in \mathrm{B}$ then $\mathrm{Xn} \in$ Type, and if the $, \mathrm{Xi}, \mathrm{Xj}$ is not belong to the same breach,then $X i$ exclude $X j, n \geqslant i \geqslant 1, n$ $\geqslant j \geqslant 1 ;(2)$ If $X i \in B$ and $B \neq \varnothing$, then $X i \rightarrow B, n \geqslant i \geqslant 1$;

- $P($ Parallel Node)is a collection, $P=\{X 1$, $X 2 \ldots, X n$ and $n \geqslant 2$,if $X n \in P$ then $X n \in T y p e$;

- $\mathrm{Rn}($ root node)is a collection, $\mathrm{Rn}=\{X 1$, $\mathrm{X} 2 \ldots, \mathrm{Xn}\}$ and $\mathrm{Rn} \neq \varnothing$,if $\mathrm{Xn} \in \mathrm{Rn}$ then $\mathrm{Xn} \in \mathrm{Type}$;

- $\mathrm{L}(\mathrm{Loop}$ node) is a collection, $\mathrm{L}=\{\mathrm{X} 1$, $\mathrm{X} 2 \ldots, \mathrm{Xn}\}$, if $\mathrm{Xn} \in \mathrm{L}, \mathrm{Xn} \in \mathrm{Type}$ and $\mathrm{L} \neq \varnothing$ then $\mathrm{Xi}$ $\leftrightarrow X j, n \geqslant i \geqslant 1, n \geqslant j \geqslant 1$;

- $S$ (Sequence node)is a collection $S=\{X 1$, $\mathrm{X} 2 \ldots, \mathrm{Xn}\}$,if $\mathrm{Xn} \in \mathrm{S}$ then $\mathrm{Xn} \in \mathrm{Type}$ and $\mathrm{S} \neq \varnothing$;

3) Define status $=\{S 0, S 1, S f, S v, S n\}$. the detail explanation as the table. 
TABLE I. THE STATUS OF TASK NODES

\begin{tabular}{|l|l|l|}
\hline number & Node status & \multicolumn{1}{c|}{ Meaning } \\
\hline 1 & S0 & has not been started \\
\hline 2 & S1 & being compiled \\
\hline 3 & Sf & Approved by the completion status \\
\hline 4 & Sn & Will not be executed \\
\hline 5 & Sv & Dat a changes \\
\hline
\end{tabular}

And the relation of push as Fig. 1

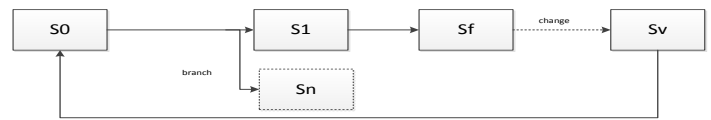

Figure 1. The relation of node push

4) The fundamental Categories of relation and its character.

- Dependency: For two nodes, namely A and B, if data results of $\mathrm{A}$ depend on that of $\mathrm{B}$, denoting as A->B.and When A $->B$, if data of A changed, then $\mathrm{B}$ is affected and state transition of $\mathrm{B}$ happens.(1). Transitivity of data dependency: If $\mathrm{A}->\mathrm{B}$ and $\mathrm{B}-$ $>C$, then $A->C(2)$. Mutual dependency: If $A->B$ and $\mathrm{B}->\mathrm{A}$, then $\mathrm{A}$ and $\mathrm{B}$ are dependent on each other, denoting as $\mathrm{A}<->\mathrm{B}$.

- Exclusive:for two nodes, namely $A$ and B,if executing A will lead to not execute B,denoting as A exclude B.And when A exclude B, if data of A changed,then $B$ is not affected and state transition of B happens.(1)if A exclude B then B exclude $\mathrm{A}$;(2)if A exclude Band $\mathrm{B} \rightarrow \mathrm{C}$, then A exclude $\mathrm{C}$.

5) Formula operation of relationship between nodes

- Define the physical dependency between nodes as $\vec{P}=\left[\begin{array}{c}p_{1} \\ p_{2} \\ \vdots \\ p_{n}\end{array}\right]$

Define the logical dependency

$\vec{L}=\left[\begin{array}{c}l_{1} \\ l_{2} \\ \vdots \\ l_{n}\end{array}\right]_{\text {,Define the correlative }}$

$\vec{U}=\left[\begin{array}{c}u_{1} \\ u_{2} \\ \vdots \\ u_{n}\end{array}\right]_{\text {,And the }}$ operation of correlation between nodes can be depict as

$$
\vec{U}=F(\vec{P}, \vec{L})
$$

$$
\begin{aligned}
& \vec{A}=\left[\begin{array}{c}
a_{1} \\
a_{2} \\
\vdots \\
a_{m}
\end{array}\right]_{\text {and }} \quad \vec{B}=\left[\begin{array}{c}
b_{1} \\
b_{2} \\
\vdots \\
b_{m}
\end{array}\right]_{\text {then }} \\
& \qquad(\overrightarrow{\boldsymbol{A}}, \overrightarrow{\boldsymbol{B}})=\left[\begin{array}{c}
a_{1} \vee b_{1} \\
\boldsymbol{a}_{2} \vee \boldsymbol{b}_{2} \\
\vdots \\
\boldsymbol{a}_{m} \vee \boldsymbol{b}_{m}
\end{array}\right]
\end{aligned}
$$

the symbol $\vee$ is or operation。

\section{B. The algorithms of model}

TR-VC mainly aim to identify the range of version change and restart the workflow. TR-VC is based on the model of workflow which is presented as a tree and the push sequence of the workflow is represented by the node number and the node implementation rely on the NS transformed. TR-VC mainly involve two algorithms, one is RWN and the other is RSW.

According to the mutual relationship between nodes, the RWN identify the range of the change and then restructure the instance of workflow.

In the algorithm, there are some nouns need to define:(1)Change state Sv: when the node A need to change, if find all nodes which have mutual relationship with A, then put the NS of A into Sv.(2)Change intermediate state Svt: when the A need to change and still have the nodes which have mutual relationship with A, then put the NS of A into Svt.

The step of algorithm is as follows:

- Ensure the node, namely A, which need to change, and put the NS of A into Svt.

- According to the formula to find a set of the nodes which have the mutual re lationship with A. Put the NS of A into Sv and then put the NS of the nodes in the set into Svt.

- Find the father node of A, and then put the NS of father node into Svt. If can take the node as A from the set, then go to step2. Else, go to step4.

- When the NS of all nodes in the workflow does not exist Svt, and the algorithm is end.

The algorithm can be depicted as Fig. 2

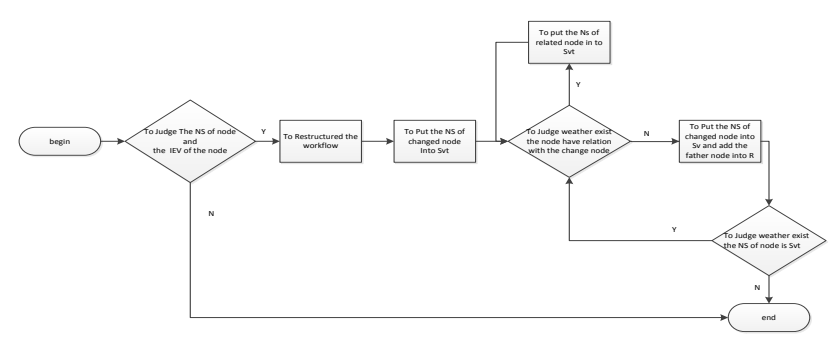

Figure 2. Algorithm:RWN

The function of RSW is restart the workflow which restructure by RWN

The mainly steps as follows: 
- According to the different NT of the change node, if can seek the proper node which NS is Sv, then execute step2. else, end.

- When complete a node, update the NS of the node and then execute the step 1.

The algorithm can be depicted as Fig. 3

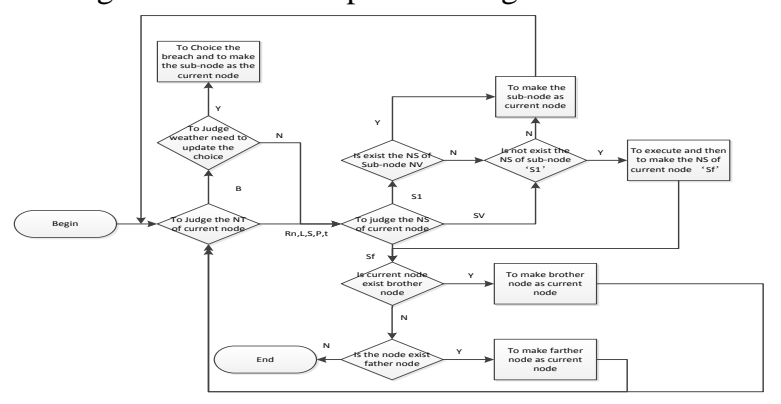

Figure 3. Algorithm:RSW

\section{IMPLEMENT ATION}

In this paper, the presented model and the presented algorithms are already implemented and applied practically. It is mainly conclude two aspects. Firstly, The workflow which execute normal is showed in Fig. 4, the green words mean the tasks are already executed, the black means not be started or not be executed which result from the choice of branch node, the red means being compiled, and the gray means need to version change. The task in the red box need to execute version change. And then the Fig. 5 show the range of the version change which seek by TRVC model.

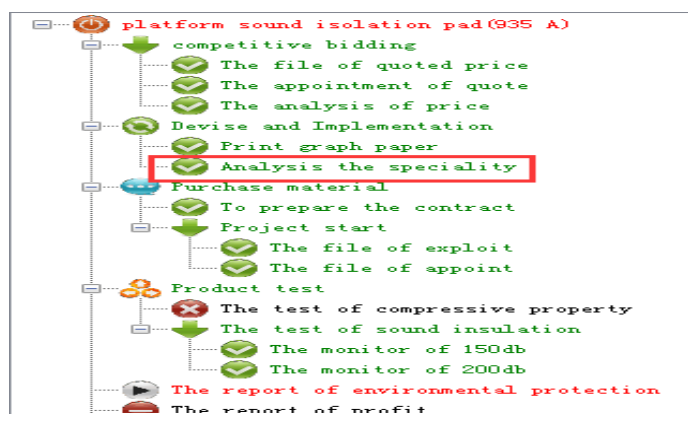

Figure 4. The normal workflow

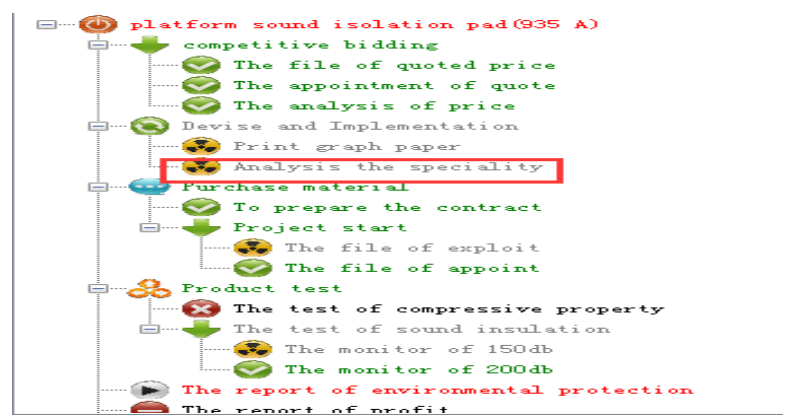

Figure 5. The version change and ensure the range

Secondly, according to the algorithms RSW, to restart the workflow. In the Fig. 6, the node in the red task is the first task need to version change and then on the basis of the nodes' sequence to execute the nodes. In the Fig. 7, the node in the red box is the last task need to execute. when the last task is completed, the version change is finished.

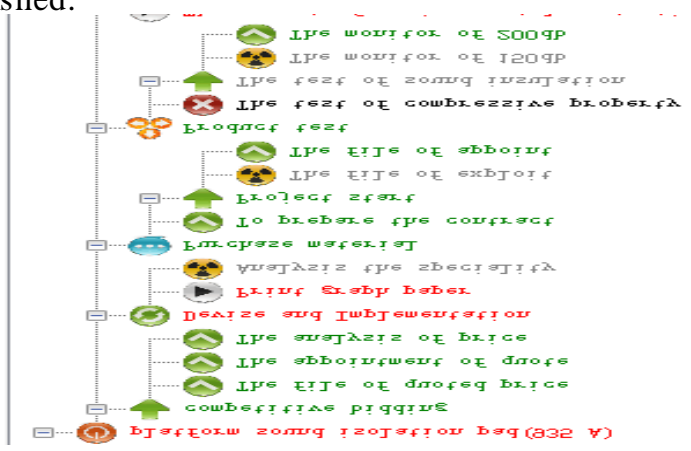

Figure 6. Execute the nodes of version change

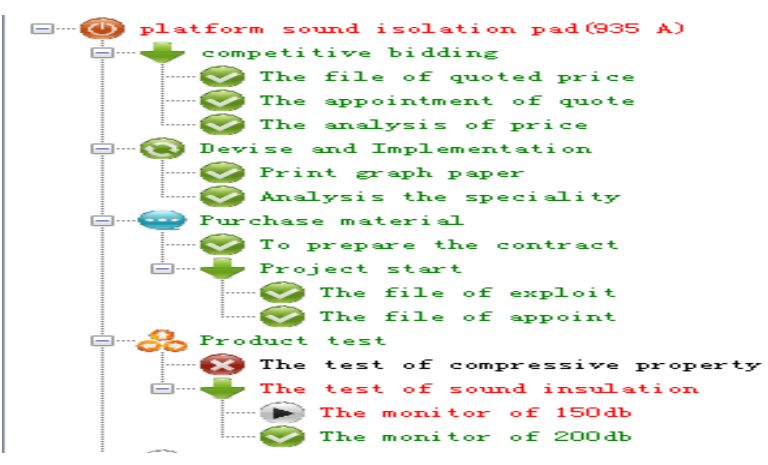

Figure 7. Complete execute

\section{CONCLUSIONS}

The TR-VC model of workflow focus on the mutual relationship between the task and aim to reduce the Reexecute the task which is unnecessary in the process of version change. In this model, it is mainly solve the two questions: the one is how to seek a smaller and more proper range of the version change; the other is to restart and to re-execute the workflow. In the future,our study should focus on the following points:

- (1)Record and analyze the times of tasks of version change, and thus forecast the possibility of change. Finally to reasonable reduce the range of version change.

- (2) On the basis of TR-VC, to calculate the cost of the version change, and then to give the more reasonable project for version change.

\section{AKNOWLEDGEMENTS}

This research is supported by National Natural Science Foundation of China under Grant No.60971089 and National Electronic Development Foundation of China under Grant No.2009537.

\section{REFERENCES}

[1] Wei Liu, et al. Design of Flexible Workflow Engine Based on .NET[C]. Advanced Materials Research Vols, 2013-08, 17761781 .

[2] Jintao Fang, et al.The data driven of workflow managment in PDM[J]. Computer-Aided Design and Manufacturing, 2002, 01: 66-68

[3] Nicholls $\mathrm{K}$, et al. Getting engineering changes under control[J]. Engng Des, 1990, 1(1): 1-6.

[4] K. G. Mcintosh. Engineering DATA Management: A Guide to Successful Implementation. McGraw-Hill, 1995, 279 pages. 
[5] Dounia Habhouba, Soumaya Cherkaoui, Alain Desrochers. Decision-Making Assistance in Engineering-Change Management Process. [J]. IEEE Transactions on Systems, Man, and Cybernetics, Part C,201 1, 41

[6] Claudia Eckert, P. John Clarkson, Winfried Zanker. Change and customisation in complex engineering domains. Research in Engineering Design - RES ENG DESIGN ,2004, vol. 15, no. 1, pp.1-21.
[7] Yang Yu, Yuanning Liu, Meng Li. Design of Flexible Workflow Engine Based on .NET[C].2013International Conference on Measurement and Control Engineering (ICMCE 2013), 2013-08, 1776-1781.

[8] Fei He, et al. Workflow Model Design based on N-tree for Process Management in PDM[C].Advanced Materials Research Vols, 2011, 268-270. 